PROCEEDINGS OF THE

AMERICAN MATHEMATICAL SOCIETY

Volume 140, Number 7, July 2012, Pages 2513-2526

S 0002-9939(2011)11093-1

Article electronically published on November 8, 2011

\title{
SHARP WEAK TYPE INEQUALITIES FOR THE HAAR SYSTEM AND RELATED ESTIMATES FOR NONSYMMETRIC MARTINGALE TRANSFORMS
}

\author{
ADAM OSȨKOWSKI
}

(Communicated by Richard C. Bradley)

Abstract. For any $1 \leq p<\infty$, we determine the optimal constant $C_{p}$ such that the following holds. If $\left(h_{k}\right)_{k>0}$ is the Haar system, then for any vectors $a_{k}$ from a separable Hilbert space $\mathcal{H}$ and $\theta_{k} \in\{0,1\}, k=0,1,2, \ldots$, we have

$$
\left\|\sum_{k=0}^{n} \theta_{k} a_{k} h_{k}\right\|_{p, \infty} \leq C_{p}\left\|\sum_{k=0}^{n} a_{k} h_{k}\right\|_{p}
$$

This is generalized to the weak type inequality

$$
\|g\|_{p, \infty} \leq C_{p}\|f\|_{p}
$$

where $f$ is an $\mathcal{H}$-valued martingale and $g$ is its transform by a predictable sequence taking values in $[0,1]$. We extend this further to the estimate

$$
\|Y\|_{p, \infty} \leq C_{p}\|X\|_{p},
$$

valid for any two $\mathcal{H}$-valued continuous-time martingales $X, Y$, such that $\left([Y, X-Y]_{t}\right)$ is nondecreasing and nonnegative as a function of $t$.

\section{INTRODUCTION}

Let $1<p<\infty$ and let $\left(h_{k}\right)_{k \geq 0}$ be the Haar system in $L^{p}$. In his classical result, Marcinkiewicz [9] proved that there is a universal finite constant $c_{p}$ such that

$$
c_{p}^{-1}\left\|\sum_{k=0}^{n} a_{k} h_{k}\right\|\left\|_{p} \leq\right\| \sum_{k=0}^{n} \varepsilon_{k} a_{k} h_{k}\|\|_{p} \leq c_{p}\left\|\sum_{k=0}^{n} a_{k} h_{k}\right\|_{p}
$$

for any $n$ and any $a_{k} \in \mathbb{R}, \varepsilon_{k} \in\{-1,1\}, k=0,1,2, \ldots, n$. This result was extended by Burkholder [1] to the martingale setting. Let $(\Omega, \mathcal{F}, \mathbb{P})$ be a probability space, filtered by $\left(\mathcal{F}_{k}\right)_{k \geq 0}$, a nondecreasing family of sub- $\sigma$-fields of $\mathcal{F}$. Let $f=\left(f_{k}\right)_{k \geq 0}$ be a real-valued martingale with the difference sequence $\left(d f_{k}\right)_{k \geq 0}$ given by $d f_{0}=f_{0}$ and $d f_{k}=f_{k}-f_{k-1}$ for $k \geq 1$. Let $g$ be a transform of $f$ by a real predictable sequence $v=\left(v_{k}\right)_{k \geq 0}$ bounded in absolute value by 1: that is, $d g_{k}=v_{k} d f_{k}$ for all $k \geq 0$. By predictability we mean that each term $v_{k}$ is measurable with respect to

Received by the editors July 8, 2010 and, in revised form, February 21, 2011.

2010 Mathematics Subject Classification. Primary 60G42; Secondary 60G44.

Key words and phrases. Martingale, martingale transform, differential subordination, weak type inequality, unconditional constant, Haar system.

Partially supported by MNiSW Grant N N201 397437. 
$\mathcal{F}_{(k-1) \vee 0}$. Then (cf. [1]) for $1<p<\infty$ there is an absolute constant $c_{p}^{\prime}$ for which

$$
\|g\|_{p} \leq c_{p}^{\prime}\|f\|_{p}
$$

Here we have used the notation $\|f\|_{p}=\sup _{n}\left\|f_{n}\right\|_{p}$. Let $c_{p}(1.1), c_{p}^{\prime}(1.2)$ denote the optimal constants in (1.1) and (1.2), respectively. The Haar system is a martingale difference sequence with respect to its natural filtration (with the probability space being the Lebesgue unit interval) and hence so is $\left(a_{k} h_{k}\right)_{k \geq 0}$, for given fixed real numbers $a_{0}, a_{1}, a_{2}, \ldots$ Therefore, $c_{p}(1.1) \leq c_{p}^{\prime}(1.2)$ for all $1<p<\infty$. It follows from the results of Burkholder [2] and Maurey [10] that in fact the constants coincide: $c_{p}(1.1)=c_{p}^{\prime}(1.2)$ for all $1<p<\infty$. The question about the precise value of $c_{p}(1.1)$ was answered by Burkholder in [3]: $c_{p}$ (1.1) $=p^{*}-1$ (where $p^{*}=$ $\max \{p, p /(p-1)\})$ for $1<p<\infty$. Furthermore, the constant does not change if we allow the martingales and the terms $a_{k}$ to take values in a separable Hilbert space $\mathcal{H}$. This determines the complex unconditional basis constant of the Haar system:

$$
\sup \left\{\left\|\sum_{k=0}^{n} \varepsilon_{k} a_{k} h_{k}\right\|_{p}\right\}=p^{*}-1, \quad 1<p<\infty,
$$

where the supremum is taken over all $n$, all sequences $\varepsilon_{0}, \varepsilon_{1}, \varepsilon_{2}, \ldots$ of signs and all complex numbers $a_{0}, a_{1}, a_{2}, \ldots$ satisfying $\left\|\sum_{k=0}^{n} a_{k} h_{k}\right\|_{p}=1$ (cf. [5]).

For $p=1$ the inequalities (1.1) and (1.2) do not hold with any finite constant, but one can establish a corresponding weak type estimate. Burkholder [3] proved the following sharp bound, for a wider range of parameters: if $1 \leq p \leq 2$, then

$$
\|g\|_{p, \infty} \leq\left(\frac{2}{\Gamma(p+1)}\right)^{1 / p}\|f\|_{p}
$$

where $\|g\|_{p, \infty}=\sup _{\lambda>0} \lambda\left(\mathbb{P}\left(\sup _{n}\left|g_{n}\right| \geq \lambda\right)\right)^{1 / p}$. For $p>2$, Suh [12] showed that

$$
\|g\|_{p, \infty} \leq\left(p^{p-1} / 2\right)^{1 / p}\|f\|_{p} .
$$

Both (1.3), (1.4) remain sharp for the Haar system, even for $\mathcal{H}$-valued coefficients. In fact, all the martingale inequalities above are valid under the less restrictive assumption of differential subordination and can be extended to the continuous-time setting. Suppose that $(\Omega, \mathcal{F}, \mathbb{P})$ is complete and equip it with a right-continuous filtration $\left(\mathcal{F}_{t}\right)_{t \geq 0}$. Let $X, Y$ be two adapted cadlag martingales taking values in $\mathcal{H}$; with no loss of generality we assume, from now on, that $\mathcal{H}=\ell^{2}$. Following [13], we say that $Y$ is differentially subordinate to $X$, if the process $\left([X, X]_{t}-[Y, Y]_{t}\right)_{t \geq 0}$ is nondecreasing and nonnegative as a function of $t$. Here $[X, Y]=\sum_{j=0}^{\infty}\left[X^{j}, Y^{\bar{j}}\right]$, where $X^{j}, Y^{j}$ stand for the $j$-th coordinates of $X$ and $Y$, respectively, and $\left[X^{j}, Y^{j}\right]$ is the quadratic covariance process of $X^{j}$ and $Y^{j}$ (see e.g. Dellacherie and Meyer [7). If we treat the discrete-time martingales $f=\left(f_{k}\right)_{k=0}^{\infty}, g=\left(g_{k}\right)_{k=0}^{\infty}$ as continuous-time processes (by $X_{t}=f_{\lfloor t\rfloor}$ and $Y_{t}=g_{\lfloor t\rfloor}$ for $t \geq 0$ ), then the above condition reads

$$
\left|d g_{k}\right| \leq\left|d f_{k}\right| \quad \text { for } k \geq 0
$$

which is the original definition of the differential subordination due to Burkholder 3. Clearly, this condition is satisfied by the martingale transforms studied above. Thus the following theorem (cf. [12, 13]) generalizes the previous inequalities (1.2), (1.3) and (1.4). We use the notation $\|X\|_{p}=\sup _{t}\left\|X_{t}\right\|_{p}$ and $\|X\|_{p, \infty}=$ $\sup _{\lambda>0} \lambda\left(\mathbb{P}\left(\sup _{t}\left|X_{t}\right| \geq \lambda\right)\right)^{1 / p}$, analogous to that of the discrete-time setting. 
Theorem 1.1. If $Y$ is differentially subordinate to $X$, then

$$
\begin{aligned}
\|Y\|_{p} & \leq c_{p}\|X\|_{p}, \quad 1<p<\infty, \\
\|Y\|_{p, \infty} & \leq\left(\frac{2}{\Gamma(p+1)}\right)^{1 / p}\|X\|_{p}, \quad 1 \leq p \leq 2, \\
\|Y\|_{p, \infty} & \leq\left(\frac{p^{p-1}}{2}\right)^{1 / p}\|X\|_{p}, \quad 2 \leq p<\infty,
\end{aligned}
$$

and the inequalities are sharp.

Let us now turn to the nonsymmetric case, a setting we will be particularly interested in. An alternative way of defining the unconditional basis constant is

$$
\sup \left\{\left\|\sum_{k \in J} a_{k} h_{k}\right\|_{p}\right\}
$$

where the supremum is taken over all $n$, all subsets $J \subseteq\{0,1,2, \ldots, n\}$ and all complex numbers $a_{0}, a_{1}, a_{2}, \ldots, a_{n}$ satisfying $\left\|\sum_{k=0}^{n} a_{k} h_{k}\right\|_{p}=1$ (see e.g. [8]). This leads to another natural transformation of Haar series: to throw out some of its terms instead of changing their signs. In other words, it suggests that we consider in (1.1) the case when each $\varepsilon_{k}$ takes values in $\{0,1\}$. Under this nonsymmetric assumption, Choi [6] found the best constant $c_{p}^{\prime \prime}$ in (1.1) for real coefficients $a_{0}, a_{1}, a_{2}, \ldots$ He also showed that if a martingale $f$ is real and each $v_{k}$ takes values in $[0,1]$, then (1.2) holds with the same constant $c_{p}^{\prime \prime}$. Since the description of the constant is quite complicated, we do not present it here and refer the interested reader to [6].

There is a natural question about the best constants in the corresponding weak type estimates for the Haar system and the extension of these bounds to continuoustime martingales. We will study this problem in the general case when the coefficients $a_{0}, a_{1}, a_{2}, \ldots$ as well as the processes take values in a Hilbert space $\mathcal{H}$. The role of "non-symmetric differential subordination" is played by the condition

$$
\left([X, Y]_{t}-[Y, Y]_{t}\right) \text { is nonnegative and nondecreasing as a function of } t .
$$

This generalizes nonsymmetric martingale transforms: assume that $f$ is a martingale and $g$ is its transform by a predictable sequence $v$. If we treat these as continuous-time processes, we see that the condition (1.6) reads $\left(v_{k}-v_{k}^{2}\right) d f_{k}^{2} \geq 0$ for all $k$, and hence it is satisfied if the variables $v_{k}$ take values in $[0,1]$.

We turn to the formulation of our main result. Let

$$
C_{p}= \begin{cases}1 & \text { if } 1 \leq p \leq 2, \\ \frac{1}{2}\left[\frac{(2 c+p-1)^{p-1}}{c+1}\right]^{1 / p} & \text { if } p>2,\end{cases}
$$

where $c=c(p)>1$ is the unique positive number satisfying

$$
c^{p-1}=2 c+1 .
$$

Theorem 1.2. Let $X, Y$ be two Hilbert-space-valued martingales satisfying (1.6). Then for any $1 \leq p<\infty$ we have

$$
\|Y\|_{p, \infty} \leq C_{p}\|X\|_{p}
$$


and the constant $C_{p}$ is the best possible. It is already the best possible in the following one-sided bound for the Haar system:

$$
\left|\left\{r \in[0,1]: \sum_{k=0}^{n} \theta_{k} a_{k} h_{k}(r) \geq 1\right\}\right| \leq C_{p}^{p}\left\|\sum_{k=0}^{n} a_{k} h_{k} \mid\right\|_{p}^{p}
$$

for all $n, a_{k} \in \mathbb{R}$ and $\theta_{k} \in\{0,1\}, k=0,1,2, \ldots, n$.

A few words about the proof and the organization of the paper. Our approach is based on Burkholder's technique, which exploits special functions which have certain convex type properties. To be more precise, the inequality (1.8) reduces to $\mathbb{E} V_{p}\left(X_{t}, Y_{t}\right) \leq 0$ for some appropriate function $V_{p}: \mathcal{H} \times \mathcal{H} \rightarrow \mathbb{R}$ and all $t \geq 0$. The key to study this inequality is to find a majorant $U_{p}$ of $V_{p}$ such that $\left(U_{p}\left(X_{t}, Y_{t}\right)\right)_{t \geq 0}$ is an $\left(\mathcal{F}_{t}\right)$-supermartingale satisfying $U_{p}\left(X_{0}, Y_{0}\right) \leq 0$. This is the way we prove Theorem 1.2 for $1 \leq p \leq 2$; see Section 2 below. For $p>2$ our argument turns out to be substantially different and uses an "integration trick" developed by the author in 11. First we show that $\mathbb{E} U_{\infty}\left(X_{t}, Y_{t}\right) \leq 0$ for some simple $U_{\infty}: \mathcal{H} \times \mathcal{H} \rightarrow \mathbb{R}$ and all $t \geq 0$, and then complicate the function by integrating it against a certain positive kernel, thus obtaining the appropriate majorant; see Section 3. The final part of the paper contains the proof of a technical fact needed in the earlier considerations.

\section{The CASE $1 \leq p \leq 2$}

The main object in this section is the function $U_{p}: \mathcal{H} \times \mathcal{H} \rightarrow \mathbb{R}$ given by

$$
U_{p}(x, y)= \begin{cases}p y \cdot(y-x) & \text { if }|x|+|2 y-x|<2, \\ p-p|x| & \text { if }|x|+|2 y-x| \geq 2 .\end{cases}
$$

Here and below, the dot - denotes the scalar product in $\mathcal{H}$ and $|x|$ stands for the norm of $x \in \mathcal{H}$. Let $V_{p}: \mathcal{H} \times \mathcal{H} \rightarrow \mathbb{R}$ be defined by

$$
V_{p}(x, y)=1_{\{|y| \geq 1\}}-|x|^{p} .
$$

We have the following majorization.

Lemma 2.1. For all $x, y \in \mathcal{H}$ we have

$$
U_{p}(x, y) \geq V_{p}(x, y) .
$$

Proof. If $|x|+|2 y-x|<2$, then $|y| \leq|x / 2|+|y-x / 2|<1$ and, consequently,

$$
1_{\{|y| \geq 1\}}-|x|^{p}=-|x|^{p} \leq-\frac{p|x|^{2}}{4} \leq p|y|(|y|-|x|) \leq p y \cdot(y-x) .
$$

On the other hand, if $|x|+|2 y-x| \geq 2$, then (2.1) follows immediately from the estimate $p-p s \geq 1-s^{p}$, valid for all $s \geq 0$, by virtue of the mean-value theorem.

Lemma 2.2. Suppose that martingales $X, Y$ satisfy the condition (1.6). Then for any $t \geq 0$ we have

$$
\mathbb{E} U_{p}\left(X_{t}, Y_{t}\right) \leq 0 .
$$

Proof. Let $\mathcal{U}: \mathcal{H} \times \mathcal{H} \rightarrow \mathbb{R}$ be given by

$$
\mathcal{U}(x, y)= \begin{cases}|y|^{2}-|x|^{2} & \text { if }|x|+|y|<1 \\ 1-2|x| & \text { if }|x|+|y| \geq 1\end{cases}
$$


This is Burkholder's special function corresponding to the weak type inequality (1.3) for $p=1$ (cf. [3]). As shown by Wang (see the proof of Theorem 3 in [13]), if $\zeta=\left(\zeta_{t}\right)$ is differentially subordinate to $\xi=\left(\xi_{t}\right)$, then for any $t \geq 0$ we have

$$
\mathbb{E} \mathcal{U}\left(\zeta_{t}, \xi_{t}\right) \leq 0 \text {. }
$$

We apply this to the martingales $\xi=X / 2$ and $\zeta=-X / 2+Y$; the differential subordination follows from the identity

$$
[X / 2, X / 2]_{t}-[-X / 2+Y,-X / 2+Y]_{t}=[X, Y]_{t}-[Y, Y]_{t} .
$$

The proof is completed by noting that $U_{p}(x, y)=p \mathcal{U}(x / 2,-x / 2+y)$ for all $x, y$.

Now we turn to the proof of Theorem 1.2 in the case $1 \leq p \leq 2$.

Proof of Theorem 1.2. Obviously, the constant $C_{p}=1$ is the best possible in (1.9): take $a_{0}=\theta_{0}=1$ and $a_{k}=\theta_{k}=0$ for $k \geq 1$. Therefore all we need is to establish the estimate (1.8). Note that we may assume that $X$ is bounded in $L^{p}$; otherwise there is nothing to prove. By homogeneity, we will be done if we show that

$$
\mathbb{P}\left(Y^{*} \geq 1\right) \leq\|X\|_{p}^{p}
$$

where $Y^{*}=\sup _{t \geq 0}\left|Y_{t}\right|$ is the maximal function of $Y$. Observe that by virtue of (2.1) and (2.2) we have

$$
\mathbb{P}\left(\left|Y_{t}\right| \geq 1\right) \leq \mathbb{E}\left|X_{t}\right|^{p} \quad \text { for } t \geq 0 .
$$

Now take $\varepsilon \in(0,1)$ and introduce the stopping time $\tau=\inf \left\{s \geq 0:\left|Y_{s}\right| \geq 1-\varepsilon\right\}$. We have that

$$
\left\{Y^{*} \geq 1\right\} \subset\left\{\left|Y_{t}\right| \geq 1-\varepsilon \text { for some } t\right\}=\left\{\left|Y_{\tau \wedge t}\right| \geq 1-\varepsilon \text { for some } t\right\} .
$$

Since the family $\left(\left\{\left|Y_{\tau \wedge s}\right| \geq 1-\varepsilon\right\}\right)_{s}$ is nondecreasing and

$$
\left\{\left|Y_{\tau \wedge t}\right| \geq 1-\varepsilon \text { for some } t\right\}=\bigcup_{t \geq 0}\left\{\left|Y_{\tau \wedge t}\right| \geq 1-\varepsilon\right\},
$$

we get $\mathbb{P}\left(Y^{*} \geq 1\right) \leq \lim _{t \rightarrow \infty} \mathbb{P}\left(Y_{\tau \wedge t} \geq 1-\varepsilon\right)$. Now it is easy to see that the pair $\left(X_{\tau \wedge t} /(1-\varepsilon)\right),\left(Y_{\tau \wedge t} /(1-\varepsilon)\right)$ satisfies (1.6). Applying (2.4) to this pair gives

$$
\lim _{t \rightarrow \infty} \mathbb{P}\left(Y_{\tau \wedge t} \geq 1-\varepsilon\right) \leq(1-\varepsilon)^{-p} \mathbb{E}\left|X_{\tau \wedge t}\right|^{p} \leq(1-\varepsilon)^{-p}\|X\|_{p}^{p} .
$$

Thus (2.3) follows, since $\varepsilon$ was arbitrary.

\section{THE CASE $p>2$}

This is more involved. Define an auxiliary function $U_{\infty}: \mathcal{H} \times \mathcal{H} \rightarrow \mathbb{R}$ by

$$
U_{\infty}(x, y)= \begin{cases}0 & \text { if }|x|+|2 y-x|<1, \\ (|2 y-x|-1)^{2}-|x|^{2} & \text { if }|x|+|2 y-x| \geq 1 .\end{cases}
$$

Later on, we will need the following properties of this function.

Lemma 3.1. (i) There is an absolute constant $A>0$ such that for all $x, y \in \mathcal{H}$,

$$
U_{\infty}(x, y) \leq A\left(|x|^{2}+|y|^{2}+1\right)
$$

(ii) For all $x, y \in \mathcal{H}$ we have

$$
U_{\infty}(x, y) \leq(|2 y-x|-1)^{2}-|x|^{2} .
$$

(iii) If $x, y, h, k \in \mathcal{H}$ satisfy

$$
|x|+|2 y-x| \leq 1, \quad|x+h|+|2(y+k)-(x+h)| \geq 1
$$


and

$$
|2 k-h| \leq|h|,
$$

then $U_{\infty}(x+h, y+k) \leq 0$.

(iv) If $x, y \in \mathcal{H}$ satisfy $x \cdot y-|y|^{2} \geq 0$, then $U_{\infty}(x, y) \leq 0$.

Proof. (i), (ii) These inequalities are evident from the very definition of $U_{\infty}$.

(iii) The desired inequality can be written in the form

$$
-|x+h| \leq|2(y+k)-(x+h)|-1 \leq|x+h| .
$$

The left inequality is precisely the second condition in (3.3). To get the right one, note that by a triangle inequality, (3.3) and (3.4),

$|2(y+k)-(x+h)|-1 \leq|2 y-x|+|2 k-h|-1 \leq|2 y-x|-1+|h| \leq-|x|+|h| \leq|x+h|$.

(iv) The estimate is trivial if $|x|+|2 y-x| \leq 1$. If the reverse holds, note that $x \cdot y-|y|^{2} \geq 0$ is equivalent to $|x|^{2} \geq|2 y-x|^{2}$ and hence

$$
U_{\infty}(x, y)=(|2 y-x|+|x|-1)(|2 y-x|-|x|-1) \leq 0 .
$$

The next result is a dual version of Lemma 2.2 .

Lemma 3.2. Suppose that the martingales $X, Y$ are bounded in $L^{2}$ and satisfy the condition (1.6). Then for any $t \geq 0$,

$$
\mathbb{E} U_{\infty}\left(X_{t}, Y_{t}\right) \leq 0 \text {. }
$$

Proof. First note that by (3.1), the random variable $U_{\infty}\left(X_{t}, Y_{t}\right)$ is integrable. Let $\tau=\inf \left\{s \geq 0:\left|X_{s}\right|+\left|2 Y_{s}-X_{s}\right|>1\right\}$. We will show the following three statements:

$$
\begin{gathered}
\mathbb{E} U_{\infty}\left(X_{t}, Y_{t}\right) 1_{\left\{\left|X_{0}\right|+\left|2 Y_{0}-X_{0}\right|>1\right\}} \leq \mathbb{E} U_{\infty}\left(X_{0}, Y_{0}\right) 1_{\left\{\left|X_{0}\right|+\left|2 Y_{0}-X_{0}\right|>1\right\}}, \\
U_{\infty}\left(X_{t}, Y_{t}\right)=U_{\infty}\left(X_{0}, Y_{0}\right)=0 \quad \text { on }\left\{\left|X_{0}\right|+\left|2 Y_{0}-X_{0}\right| \leq 1, \tau>t\right\}
\end{gathered}
$$

and

$$
\mathbb{E} U_{\infty}\left(X_{t}, Y_{t}\right) 1_{\left\{\left|X_{0}\right|+\left|2 Y_{0}-X_{0}\right| \leq 1, \tau \leq t\right\}} \leq \mathbb{E} U_{\infty}\left(X_{0}, Y_{0}\right) 1_{\left\{\left|X_{0}\right|+\left|2 Y_{0}-X_{0}\right| \leq 1, \tau \leq t\right\}}
$$

These three facts yield the claim: indeed, they give $\mathbb{E} U\left(X_{t}, Y_{t}\right) \leq \mathbb{E} U\left(X_{0}, Y_{0}\right)$, and it suffices to note that $U\left(X_{0}, Y_{0}\right) \leq 0$ in view of (1.6) and part (iv) of Lemma 3.1.

To prove (3.6), use (3.2) to get

$$
\mathbb{E}\left[U_{\infty}\left(X_{t}, Y_{t}\right) \mid \mathcal{F}_{0}\right] \leq 4 \mathbb{E}\left[Y_{t} \cdot\left(Y_{t}-X_{t}\right) \mid \mathcal{F}_{0}\right]-2 \mathbb{E}\left(\left|2 Y_{t}-X_{t}\right| \mid \mathcal{F}_{0}\right)+1
$$

Clearly, $\mathbb{E}\left(\left|2 Y_{t}-X_{t}\right| \mid \mathcal{F}_{0}\right) \geq\left|2 Y_{0}-X_{0}\right|$. Moreover, by (1.6), we have that $\mathbb{E}\left[Y_{t}\left(Y_{t}-X_{t}\right)-Y_{0}\left(Y_{0}-X_{0}\right) \mid \mathcal{F}_{0}\right]=-\mathbb{E}\left[\left([X, Y]_{t}-[Y, Y]_{t}\right)-\left([X, Y]_{0}-[Y, Y]_{0}\right) \mid \mathcal{F}_{0}\right]$ is nonpositive. Consequently, on the set $\left\{\left|X_{0}\right|+\left|2 Y_{0}-X_{0}\right|>1\right\}$,

$$
\mathbb{E}\left[U_{\infty}\left(X_{t}, Y_{t}\right) \mid \mathcal{F}_{0}\right] \leq 4 Y_{0} \cdot\left(Y_{0}-X_{0}\right)-2\left|2 Y_{0}-X_{0}\right|+1=U_{\infty}\left(X_{0}, Y_{0}\right)
$$

and (3.6) follows. The condition (3.7) is obvious, by the definition of $U_{\infty}$ and $\tau$. To get (3.8), we proceed as previously: by (3.2) and (1.6) we have, on the set $\left\{\left|X_{0}\right|+\left|2 Y_{0}-X_{0}\right| \leq 1, \tau \leq t\right\}$,

$$
\begin{aligned}
\mathbb{E}\left[U_{\infty}\left(X_{t}, Y_{t}\right) \mid \mathcal{F}_{\tau}\right] & =4 \mathbb{E}\left[Y_{t} \cdot\left(Y_{t}-X_{t}\right) \mid \mathcal{F}_{\tau}\right]-2 \mathbb{E}\left(\left|2 Y_{t}-X_{t}\right| \mid \mathcal{F}_{\tau}\right)+1 \\
& \leq 4 Y_{\tau} \cdot\left(Y_{\tau}-X_{\tau}\right)-2\left|2 Y_{\tau}-X_{\tau}\right|+1 \\
& =U_{\infty}\left(X_{\tau}, Y_{\tau}\right) .
\end{aligned}
$$


Now use part (iii) of Lemma 3.1 with $x=X_{\tau-}, y=Y_{\tau-}, h=\Delta X_{\tau}$ and $k=\Delta Y_{\tau}$ : the condition (3.3) follows from the definition of $\tau$, while (3.4) is a consequence of (1.6). Thus, $U_{\infty}\left(X_{\tau}, Y_{\tau}\right) \leq 0=U_{\infty}\left(X_{0}, Y_{0}\right)$ and the proof is complete.

We are ready to introduce the special function $U_{p}: \mathcal{H} \times \mathcal{H} \rightarrow \mathbb{R}$, corresponding to the weak type estimate for $p>2$. Recall $c=c(p)$ given by (1.7) and let

$$
b=b(p)=\frac{2(p-1)}{2 c+p-1} .
$$

Set

$$
U_{p}(x, y)=\int_{0}^{b} t^{p-1} U_{\infty}(x / t, y / t) \mathrm{d} t .
$$

Some lengthy but straightforward calculations give that

$$
U_{p}(x, y)=\frac{2}{p(p-1)(p-2)}(|x|+|2 y-x|)^{p-1}(|2 y-x|-(p-1)|x|)
$$

if $|x|+|2 y-x| \leq b$, and

$$
U_{p}(x, y)=b^{p-2}\left[\frac{|2 y-x|^{2}-|x|^{2}}{p-2}-\frac{2 b|2 y-x|}{p-1}+\frac{b^{2}}{p}\right]
$$

for $|x|+|2 y-x|>b$. We will also need the function $V_{p}: \mathcal{H} \times \mathcal{H} \rightarrow \mathbb{R}$, given by

$$
V_{p}(x, y)=\alpha_{p}\left(C_{p}^{-p} 1_{\{|y| \geq 1\}}-|x|^{p}\right),
$$

where

$$
\alpha_{p}=\frac{2(p-1)^{p-2}}{p(p-2)}
$$

We have the following majorization.

Lemma 3.3. For all $x, y \in \mathcal{H}$, we have

$$
U_{p}(x, y) \geq V_{p}(x, y) .
$$

The justification of this estimate is quite involved, so for the sake of clarity we postpone it to Section 4 and proceed with the proof of Theorem 1.2.

Proof of (1.8). We may assume that $X$ is bounded in $L^{p}$. Then so is $Y$ : since $-X / 2+Y$ is differentially subordinate to $X / 2$ (see the proof of Lemma 2.2 above), the inequality (1.5) implies that

$$
\|Y\|_{p} \leq\|-X / 2+Y\|_{p}+\|X / 2\|_{p} \leq p^{*}\|X / 2\|_{p}<\infty .
$$

As in the case $1 \leq p \leq 2$, we reduce the desired estimate to

$$
\mathbb{P}\left(\left|Y_{t}\right| \geq 1\right) \leq C_{p}^{p}\left|X_{t}\right|^{p}, \quad t \geq 0
$$

By (3.12), this will be done if we show that $\mathbb{E} U_{p}\left(X_{t}, Y_{t}\right) \leq 0$. This follows immediately from (3.5), the definition of $U_{p}$ and Fubini's theorem. To see that the latter is applicable, note that by (3.1),

$$
\mathbb{E}\left|U_{p}\left(X_{t}, Y_{t}\right)\right| \leq A \mathbb{E}\left[\frac{b^{p-2}\left(\left|X_{t}\right|^{2}+\left|Y_{t}\right|^{2}\right)}{p-2}+\frac{b^{p}}{p}\right]<\infty,
$$

since $X, Y$ are bounded in $L^{p}$. 
We turn to the sharpness of (1.9), which is the most technical element of the paper. We will need the following fact, which relates the validity of a given inequality for the Haar system to a certain boundary value problem (for similar results, see e.g. Section 11 in [3] or Section 7 in [4]).

Lemma 3.4. Let $V: \mathbb{R} \times \mathbb{R} \rightarrow \mathbb{R}$ be a given Borel function, locally bounded from below. Assume that

$$
\int_{0}^{1} V\left(\sum_{k=0}^{n} a_{k} h_{k}(r), \sum_{k=0}^{n} a_{k} \theta_{k} h_{k}(r)\right) \mathrm{d} r \leq 0
$$

for all $n$ and all $a_{k} \in \mathbb{R}, \theta_{k} \in\{0,1\}, k=0,1,2, \ldots, n$. Then there is a function $W: \mathbb{R} \times \mathbb{R} \rightarrow \mathbb{R}$ satisfying the following properties.

(a) We have $W(x, x) \leq 0$ for any $x \in \mathbb{R}$.

(b) For all $x, y \in \mathbb{R}$ we have $W(x, y) \geq V(x, y)$.

(c) The function $W$ is concave along any line of slope 0 or 1 .

Proof. Define $W: \mathbb{R} \times \mathbb{R} \rightarrow(-\infty, \infty]$ by

$$
W(x, y)=\sup \left\{\int_{0}^{1} V\left(x+\sum_{k=1}^{n} a_{k} h_{k}(r), y+\sum_{k=1}^{n} a_{k} \theta_{k} h_{k}(r)\right) \mathrm{d} r\right\},
$$

where the supremum is taken over all $n$ and all $a_{k} \in \mathbb{R}, \theta_{k} \in\{0,1\}, k=1,2, \ldots, n$. Then the property (a) is a consequence of (3.13), while (b) follows from the definition of $W$ by considering the sequence $a_{1}=a_{2}=\ldots=0$. To get (c), we use Burkholder's "splicing" argument: take any line $L$ of slope 1 and any point $(x, y)$ lying on it. Take $d>0$, an integer $N$, sequences $a_{1}^{+}, a_{2}^{+}, \ldots, a_{N}^{+}, a_{1}^{-}, a_{2}^{-}, \ldots, a_{N}^{-}$ of real numbers and $\theta_{1}^{+}, \theta_{2}^{+}, \ldots, \theta_{N}^{+}, \theta_{1}^{-}, \theta_{2}^{-}, \ldots, \theta_{N}^{-}$with $\theta_{k}^{ \pm} \in\{0,1\}$. Let

$$
Z_{x, y}^{ \pm}(r)=\left(x \pm d+\sum_{k=1}^{N} a_{k}^{ \pm} h_{k}(r), y \pm d+\sum_{k=1}^{N} a_{k}^{ \pm} \theta_{k}^{ \pm} h_{k}(r)\right)
$$

and splice the functions $Z^{+}$and $Z^{-}$together in the following way:

$$
Z_{x, y}(r)= \begin{cases}Z_{x, y}^{-}(2 r) & \text { if } 0 \leq r \leq 1 / 2 \\ Z_{x, y}^{+}(2 r-1) & \text { if } 1 / 2<r \leq 1\end{cases}
$$

Then it is easily seen that

$$
Z_{x, y}(r)=\left(x+\sum_{k=1}^{2 N} a_{k} h_{k}(r), y+\sum_{k=1}^{2 N} a_{k} \theta_{k} h_{k}(r)\right),
$$

where for any $1 \leq k \leq 2 N$, there is $\ell \leq N$ such that $\left(a_{k}, \theta_{k}\right)=\left(a_{\ell}^{\prime}, \theta_{\ell}^{\prime}\right)$ or $\left(a_{k}, \theta_{k}\right)=$ $\left(a_{\ell}^{\prime \prime}, \theta_{\ell}^{\prime \prime}\right)$. Thus

$$
W(x, y) \geq \int_{0}^{1} V\left(Z_{x, y}(r)\right) \mathrm{d} r=\int_{0}^{1 / 2} V\left(Z_{x, y}^{-}(2 r)\right) \mathrm{d} r+\int_{1 / 2}^{1} V\left(Z_{x, y}^{+}(2 r-1)\right) \mathrm{d} r
$$

and taking the supremum over all $N, a_{k}^{ \pm}$and $\theta_{k}^{ \pm}$as above yields

$$
W(x, y) \geq(W(x-d, y-d)+W(x+d, y+d)) / 2 .
$$

Since $x, y$, and $d$ were arbitrary, $W$ is midpoint concave along $L$. Analogous arguments lead to the midpoint concavity along the lines of slope 0 . This yields the 
finiteness of $W$ : indeed, for any $x, y \in \mathbb{R}$ we have, by (a) and (b),

$$
0 \geq W(y, y) \geq \frac{1}{2} W(x, y)+\frac{1}{2} W(2 y-x, y) \geq \frac{1}{2} W(x, y)+\frac{1}{2} V(2 y-x, y),
$$

so $W(x, y) \leq-V(2 y-x, y)$. Finally, $W$ is locally bounded from below by virtue of (b) and the fact that $V$ also has this property. This, combined with the midpoint concavity of $W$ along the lines of slope 0 or 1 , yields (c).

We are ready to study (1.9). Let $p>2,0<\gamma_{p}<C_{p}$ and assume that

$$
\left|\left\{r \in[0,1]: \sum_{k=0}^{n} a_{k} \theta_{k} h_{k}(r) \geq 1\right\}\right| \leq \gamma_{p}^{p} \int_{0}^{1}\left|\sum_{k=0}^{n} a_{k} h_{k}(r)\right|^{p} \mathrm{~d} r,
$$

for all $n$ and all $a_{k} \in \mathbb{R}, \theta_{k} \in\{0,1\}, k=0,1,2, \ldots, n$. Take $\beta_{p} \in\left(\gamma_{p}, C_{p}\right)$ and

$$
V_{p}^{\beta_{p}}(x, y)=1_{\{y \geq 1\}}-\beta_{p}^{p}|x|^{p} .
$$

Let $W_{p}$ be given by (3.14), with $V=V_{p}^{\beta_{p}}$. For clarity, we split the remaining part of the proof into a few steps. Recall $b$ and $c$, given by (3.9) and (1.7), respectively.

Step 1 . The starting point is the equation

$$
W_{p}(0, y)=0 \quad \text { for sufficiently small } y>0 .
$$

To see this, note first that if $y<1$, then, by (b), $W_{p}(0, y) \geq 0$. On the other hand, let $y$ be a positive number satisfying $\beta_{p}(1-y) \geq \gamma_{p}$. Take an integer $n$, numbers $a_{1}, a_{2}, \ldots, a_{n}$ belonging to $\mathbb{R}$ and $\theta_{1}, \theta_{2}, \ldots, \theta_{n}$ belonging to $\{0,1\}$. We have

$$
\begin{aligned}
& \left|\left\{r \in[0,1]: y+\sum_{k=1}^{n} a_{k} \theta_{k} h_{k}(r) \geq 1\right\}\right|-\beta_{p}^{p} \int_{0}^{1}\left|\sum_{k=1}^{n} a_{k} h_{k}(r)\right|^{p} \mathrm{~d} r \\
& =\left|\left\{r \in[0,1]: \sum_{k=1}^{n} \frac{a_{k}}{1-y} \theta_{k} h_{k}(r) \geq 1\right\}\right|-\beta_{p}^{p}(1-y)^{p} \int_{0}^{1}\left|\sum_{k=1}^{n} \frac{a_{k}}{1-y} h_{k}(r)\right|^{p} \mathrm{~d} r \\
& \leq\left|\left\{r \in[0,1]: \sum_{k=1}^{n} \frac{a_{k}}{1-y} \theta_{k} h_{k}(r) \geq 1\right\}\right|-\gamma_{p}^{p} \int_{0}^{1}\left|\sum_{k=1}^{n} \frac{a_{k}}{1-y} h_{k}(r)\right|^{p} \mathrm{~d} r \leq 0,
\end{aligned}
$$

where the latter estimate follows from (3.15). Since $n$ and the numbers $a_{k}$ and $\theta_{k}$ were arbitrary, this gives $W_{p}(0, y) \leq 0$ and we are done.

Step 2. Note that the points

$$
P_{1}=(1-b / 2,1), P_{2}=(0, b / 2), P_{3}=(-b /(p-1), b(p-3) /(2(p-1)))
$$

lie, in this order, on a certain line of slope 1 . Moreover, by (b),

and

$$
W_{p}\left(P_{1}\right) \geq 1-\beta_{p}^{p}\left(1-\frac{b}{2}\right)^{p}=1-\beta_{p}^{p} c^{p}\left(\frac{b}{p-1}\right)^{p}
$$

Thus, combining this with (c),

$$
\begin{aligned}
W_{p}\left(P_{2}\right) & \geq \frac{\left|P_{3}-P_{2}\right|}{\left|P_{3}-P_{1}\right|} W_{p}\left(P_{1}\right)+\frac{\left|P_{2}-P_{1}\right|}{\left|P_{3}-P_{1}\right|} W_{p}\left(P_{3}\right) \\
& \geq \frac{1}{c+1}-\frac{c}{c+1} \beta_{p}^{p}\left(\frac{b}{p-1}\right)^{p}\left(1+c^{p-1}\right) .
\end{aligned}
$$


Step 3. Fix positive numbers $y$ and $\delta$. Using (c) and then (b), we get

$$
\begin{aligned}
W_{p}(0, y) & \geq \frac{\delta(p-1)}{\delta(p-1)+2} W_{p}\left(\frac{2 y}{p-1}, y\right)+\frac{2}{\delta(p-1)+2} W_{p}(-\delta y, y) \\
& \geq-\frac{\delta(p-1)}{\delta(p-1)+2} \beta_{p}^{p}\left(\frac{2 y}{p-1}\right)^{p}+\frac{2}{\delta(p-1)+2} W_{p}(-\delta y, y) .
\end{aligned}
$$

Similarly, we get

$$
W_{p}(-\delta y, y) \geq-\frac{\delta(p-1)}{2(1+\delta)} \beta_{p}^{p}\left(\frac{2 y(1+\delta)}{p-1}\right)^{p}+\frac{2(1+\delta)-\delta(p-1)}{2(1+\delta)} W_{p}(0, y(1+\delta)),
$$

and plugging this into the preceding estimate gives

$$
W_{p}(0, y) \geq A(\delta) \beta_{p}^{p} y^{p}+B(\delta) W_{p}(0, y(1+\delta))
$$

where

$$
A(\delta)=-\left(\frac{2}{p-1}\right)^{p} \frac{\delta(p-1)}{\delta(p-1)+2}\left(1+(1+\delta)^{p-1}\right)
$$

and

$$
B(\delta)=\frac{2(1+\delta)-\delta(p-1)}{(1+\delta)(\delta(p-1)+2)}
$$

One easily verifies that we have the following asymptotics:

$$
\begin{aligned}
& \lim _{\delta \rightarrow 0} \frac{A(\delta)}{\delta}=-2\left(\frac{2}{p-1}\right)^{p-1}, \\
& \lim _{\delta \rightarrow 0} \frac{B(\delta)(1+\delta)^{p-1}-1}{\delta^{2}}=0 .
\end{aligned}
$$

By induction, (3.18) leads to

$$
W_{p}(0, y) \geq \beta_{p}^{p} y^{p} A(\delta) \frac{\left[B(\delta)(1+\delta)^{p}\right]^{N}-1}{B(\delta)(1+\delta)^{p}-1}+B(\delta)^{N} W_{p}\left(0, y(1+\delta)^{N}\right)
$$

for any positive integer $N$.

Step 4. This is the final part. Take $\delta=N^{-1 / 2}, y=b(1+\delta)^{-N} / 2$, divide both sides of (3.21) by $y^{p-1}$ and let $N \rightarrow \infty$. We obtain the estimate

$$
0 \geq-b \beta_{p}^{p}\left(\frac{2}{p-1}\right)^{p-1}+\left(\frac{2}{b}\right)^{p-1} W_{p}\left(0, \frac{b}{2}\right) .
$$

To see this, note that $\delta \rightarrow 0, y \rightarrow 0$ as $N \rightarrow \infty$, and therefore, by (3.16), the lefthand side of (3.21), divided by $y^{p-1}$, converges to 0 . To deal with the right-hand side, observe that, by (3.19) and (3.20),

$$
\lim _{\delta \rightarrow 0} \frac{A(\delta)}{B(\delta)(1+\delta)^{p}-1}=\lim _{\delta \rightarrow 0} \frac{A(\delta)}{\delta B(\delta)(1+\delta)^{p-1}}=-2\left(\frac{2}{p-1}\right)^{p-1} .
$$

Furthermore, again by (3.20), we have

$$
y\left(\left[B(\delta)(1+\delta)^{p}\right]^{N}-1\right)=\frac{b}{2}\left[B(\delta)(1+\delta)^{p-1}\right]^{1 / \delta^{2}} \rightarrow \frac{b}{2}
$$

and, similarly,

$$
\frac{B(\delta)^{N}}{y^{p-1}}=\left(\frac{2}{b}\right)^{p-1}\left(B(\delta)(1+\delta)^{p-1}\right)^{1 / \delta^{2}} \rightarrow\left(\frac{2}{b}\right)^{p-1}
$$


The above three limits yield (3.22). Combining this estimate with (3.17) gives

$$
\beta_{p}^{p}\left[b\left(\frac{2}{p-1}\right)^{p-1}+\left(\frac{2}{b}\right)^{p-1} \frac{c}{c+1}\left(\frac{b}{p-1}\right)^{p}\left(1+c^{p-1}\right)\right] \geq\left(\frac{2}{b}\right)^{p-1} \frac{1}{c+1} .
$$

After some cancellations and manipulations, it can be written in the form

$$
\beta_{p}^{p} \geq \frac{(p-1)^{p}}{b^{p}(c+1)}\left[p-1+\frac{c}{c+1}\left(1+c^{p-1}\right)\right]^{-1}=C_{p}^{p}
$$

where the equality follows from (1.7). This contradicts the initial assumption $\beta_{p} \in$ $\left(\gamma_{p}, C_{p}\right)$ and completes the proof.

\section{Proof of Lemma 3.3}

We start with a reduction step: it suffices to establish the majorization in the real case and for $x, y$ satisfying $0 \leq x \leq 2 y$. To see this, let us (for a moment) write $U_{p}^{\mathcal{H}}, V_{p}^{\mathcal{H}}$ instead of $U_{p}, V_{p}$, to indicate the Hilbert space we are working with. For $x, y \in \mathcal{H}$, take $x^{\prime}=|x|$ and $y^{\prime}=|x / 2|+|x / 2-y|$. Then $0 \leq x^{\prime} \leq 2 y^{\prime}$, $2 y^{\prime}-x^{\prime}=|2 y-x|$ and $y^{\prime} \geq|y|$, so

$$
U_{p}^{\mathcal{H}}(x, y)-V_{p}^{\mathcal{H}}(x, y) \geq U_{p}^{\mathbb{R}}\left(x^{\prime}, y^{\prime}\right)-V_{p}^{\mathbb{R}}\left(x^{\prime}, y^{\prime}\right) .
$$

This justifies the reduction. We consider the cases $y \leq b / 2, y \in(b / 2,1)$ and $y \geq 1$ separately in the three lemmas below.

Lemma 4.1. We have

$$
\frac{2}{p(p-1)(p-2)}(2 y)^{p-1}(2 y-p x) \geq-\frac{2(p-1)^{p-2}}{p(p-2)} x^{p} .
$$

This yields the majorization (3.12) for $y \leq b / 2$.

Proof. The estimate is clear for $x=0$. If $x>0$ and we divide both sides by $x^{p}$, the inequality takes the form $F_{0}(2 y / x) \geq 0$, where

$$
F_{0}(s):=\frac{2}{p(p-1)(p-2)} s^{p-1}(s-p)+\frac{2(p-1)^{p-2}}{p(p-2)}
$$

for $s>0$. It suffices to note that $F_{0}$ is convex and $F_{0}(p-1)=F_{0}^{\prime}(p-1)=0$.

Lemma 4.2. (i) For all $s \geq 0$,

$$
b^{p-2}\left(-\frac{s}{p-2}+\frac{b^{2}}{p(p-1)^{2}}\right)+\frac{2(p-1)^{p-2}}{p(p-2)} s^{p / 2} \geq 0 .
$$

(ii) We have

$$
b^{p-2}\left[\frac{4 y^{2}-4 x y}{p-2}-\frac{2 b(2 y-x)}{p-1}+\frac{b^{2}}{p}\right]+\frac{2(p-1)^{p-2}}{p(p-2)} x^{p} \geq 0 .
$$

This yields the majorization (3.12) for $y \in(b / 2,1)$.

Proof. (i) Denote the left-hand side of (4.2) by $F_{1}(s)$. It is evident that the function $F_{1}$ is convex on $\mathbb{R}$. In addition, it is straightforward to check that

$$
F_{1}\left(b^{2} /(p-1)^{2}\right)=F_{1}^{\prime}\left(b^{2} /(p-1)^{2}\right)=0 .
$$

The claim follows. 
(ii) The partial derivative of the left-hand side of (4.3) with respect to $y$ equals

$$
\frac{4 b^{p-2}}{p-2}\left(2 y-x-\frac{b(p-2)}{p-1}\right) \text {. }
$$

Thus it suffices to verify the estimate for $2 y=x+b(p-2) /(p-1)$. Plug this into (4.3) to get the inequality $F_{1}\left(x^{2}\right) \geq 0$, which has already been proved in (i).

Lemma 4.3. If $y \geq 1$, then

$$
b^{p-2}\left[\frac{4 y^{2}-4 x y}{p-2}-\frac{2 b(2 y-x)}{p-1}+\frac{b^{2}}{p}\right]-\frac{2(p-1)^{p-2}}{p(p-2)}\left(\frac{1}{C_{p}^{p}}-x^{p}\right) \geq 0 .
$$

This yields the majorization (3.12) for $y \geq 1$.

Proof. We divide the proof into three parts.

Step 1. A reduction. Denoting the left-hand side of (4.5) by $F_{2}(x, y)$, we derive that its partial derivative with respect to $y$ is given by

$$
F_{2 y}(x, y)=\frac{4 b^{p-2}}{p-2}\left(2 y-x-\frac{b(p-2)}{p-1}\right) .
$$

Consequently, it suffices to establish the estimate on the line segment

$$
H_{1}=\left\{(x, y): y=1, x \geq 0,2 y-x-\frac{b(p-2)}{p-1} \geq 0\right\}
$$

and the half-line

$$
H_{2}=\left\{(x, y): y>1,2 y-x-\frac{b(p-2)}{p-1}=0\right\} .
$$

Step 2. The segment $H_{1}$. It is obvious that $x \mapsto F_{2}(x, 1)$ is convex on the interval $[0,2-b(p-2) /(p-1)]$. After some lengthy but easy calculations we verify that

$$
1-\frac{b}{2}<2-\frac{b(p-2)}{p-1} \text { and } F_{2}\left(1-\frac{b}{2}, 1\right)=F_{2}^{\prime}\left(1-\frac{b}{2}, 1\right)=0
$$

which yields the estimate on $H_{1}$.

Step 3. The half-line $H_{2}$. Plugging $2 y=x+b(p-2) /(p-1)$ into the estimate transforms it into

$$
b^{p-2}\left(-\frac{x^{2}}{p-2}+\frac{b^{2}}{p(p-1)^{2}}\right)+\frac{2(p-1)^{p-2}}{p(p-2)} x^{p} \geq \frac{2(p-1)^{p-2}}{p(p-2) C_{p}^{p}} .
$$

The left-hand side is equal to $F_{1}\left(x^{2}\right)$, where $F_{1}$ was defined in the proof of Lemma 4.2. Note that we have

$$
x \geq 2-\frac{b(p-2)}{p-1} \geq \frac{b}{p-1},
$$

the latter being equivalent to $b \leq 2$, which is obvious. Thus, by the convexity of $F_{1}$ and (4.4), we get that the left-hand side of (4.6) attains its minimum at $x=2-b(p-2) /(p-1)$. However, then the estimate reads $F_{2}(2-b(p-2) /(p-1)) \geq 0$, and we have already showed this in the preceding step. The proof is complete.

In the lemma below we show (3.12) for $y \leq-1$. 
Lemma 4.4. Let $p>2$ and $y \leq-1$.

(i) We have

$$
b^{p-1}\left[-\frac{2(p-1) y}{b}+p-2\right]-(2(p-1))^{p-1}|y|^{p-1} \leq 0 .
$$

(ii) For any $(x, y) \in \mathbb{R}^{2}$ satisfying $x \leq 2 y$ we have

$$
b^{p-2}\left[\frac{4 y^{2}-4 x y}{p-2}-\frac{2 b(2 y-x)}{p-1}+\frac{b^{2}}{p}\right]-\frac{2(p-1)^{p-2}}{p(p-2)}\left(\frac{1}{C_{p}^{p}}-|x|^{p}\right) \geq 0 .
$$

Proof. (i) Denote the left-hand side by $F_{3}(y)$. We have that

$$
F_{3}^{\prime}(y)=(2(p-1))^{p-1}\left[-\left(\frac{b}{2(p-1)}\right)^{p-2}+|y|^{p-2}(p-1)\right],
$$

and since $b /(2(p-1))=(2 c+p-1)^{-1}<1$ and $y \leq-1$, we see that the expression in the square brackets is positive. Thus $F_{3}$ is increasing and it suffices to show that $F_{3}(-1) \leq 0$. We have

$$
\begin{aligned}
F_{3}(-1) & =b^{p-1}\left[\frac{2(p-1)}{b}+p-2\right]-(2(p-1))^{p-1} \\
& =b^{p-1}\left[2 c+2 p-3-(2 c+p-1)^{p-1}\right] .
\end{aligned}
$$

It follows from a standard analysis that the function $x \mapsto x+2 p-3-(x+p-1)^{p-1}$ is decreasing on $[0, \infty)$. Using this and the trivial estimate $c \geq 1$ yields

$$
2 c+2 p-3-(2 c+p-1)^{p-1} \leq 2 p-1-(p+1)^{p-1} .
$$

It is easy to check that the right-hand side, as a function of $p \geq 2$, is decreasing and equal to 0 at 2 . This completes the proof of (4.7).

(ii) Let $y \leq-1$ be fixed and denote the left-hand side by $F_{4}(x)$. Clearly, the function $F_{4}$ is convex on $(-\infty, 2 y]$ and satisfies $F_{4}(2 y) \leq 0$, by means of Lemma 4.3 (since $F_{4}(2 y)=F_{2}(-2 y, 1)$ ). Therefore, we will be done if we show that $F_{4}^{\prime}(2 y-) \leq$ 0 . However, this inequality is equivalent to (4.7), which we have already established in (i).

\section{ACKNOWLEDGMENT}

The author would like to thank an anonymous referee for the careful reading of the first version of the paper.

\section{REFERENCES}

[1] D. L. Burkholder, Martingale transforms, Ann. Math. Statist. 37 (1966), 1494-1504. MR0208647 (34:8456)

[2] D. L. Burkholder, A geometrical characterization of Banach spaces in which martingale difference sequences are unconditional, Ann. Probab. 9 (1981), 997-1011. MR632972 (83f:60070)

[3] D. L. Burkholder, Boundary value problems and sharp inequalities for martingale transforms, Ann. Probab. 12 (1984), 647-702. MR744226 (86b:60080)

[4] D. L. Burkholder, Martingales and Fourier analysis in Banach spaces, Probability and analysis (Varrenna, 1985), Lecture Notes in Math. 1206, Springer, Berlin (1986), 61-108. MR864712(88c:42017)

[5] D. L. Burkholder, A proof of Petczyniski's conjecture for the Haar system, Studia Math. 91 (1988), 79-83. MR957287 (89j:46026) 
[6] K. P. Choi, $A$ sharp inequality for martingale transforms and the unconditional basis constant of a monotone basis in $L^{p}(0,1)$, Trans. Amer. Math. Soc. 330 (1992), 509-521. MR.1034661 (92f:60073)

[7] C. Dellacherie and P. A. Meyer, Probabilities and potential B, North-Holland, Amsterdam, 1982. MR745449 (85e:60001)

[8] R. C. James, Bases in Banach spaces, Amer. Math. Monthly 89 (1982), 625-640. MR678808 (84h:46019)

[9] J. Marcinkiewicz, Quelques théorèmes sur les séries orthogonales, Ann. Soc. Polon. Math. 16 (1937), 84-96.

[10] B. Maurey, Système de Haar, Séminaire Maurey-Schwartz (1974-1975), École Polytechnique, Paris. MR0420839 (54:8851)

[11] A. Osȩkowski, Inequalities for dominated martingales, Bernoulli 13, no. 1 (2007), 54-79. MR2307394 (2008e:60120)

[12] Y. Suh, A sharp weak type $(p, p)$ inequality $(p>2)$ for martingale transforms and other subordinate martingales, Trans. Amer. Math. Soc. 357 (2005), 1545-1564 (electronic). MR 2115376 (2005k:60134)

[13] G. Wang, Differential subordination and strong differential subordination for continuous time martingales and related sharp inequalities, Ann. Probab. 23 (1995), 522-551. MR1334160 (96b:60120)

Department of Mathematics, Informatics and Mechanics, University of Warsaw, BANACHa 2, 02-097 WARSAW, Poland

E-mail address: ados@mimuw.edu.pl 